\title{
Interpreting myocardial perfusion scintigraphy using single-photon emission computed tomography. Part 1
}

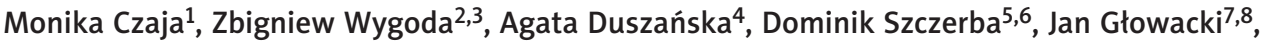 \\ Mariusz Gąsior ${ }^{9}$, Jarosław P. Wasilewski ${ }^{9}$ \\ ${ }^{1}$ Student Scientific Society, $3^{\text {rd }}$ Department of Cardiology, School of Medicine with the Division of Dentistry in Zabrze, \\ Medical University of Silesia, Katowice, Poland \\ ${ }^{2}$ Institute of Nuclear Medicine and Oncological Endocrinology, Center of Oncology, Gliwice, Poland \\ ${ }^{3}$ Laboratory of Nuclear Medicine, Silesian Center for Heart Diseases, Zabrze, Poland \\ ${ }^{4}$ Department of Cardiac Surgery, Transplantology, and Mechanical Circulatory Support, Silesian Center for Heart Diseases, \\ Zabrze, Poland \\ ${ }^{5}$ Department of Theoretical Physics, University of Silesia, Katowice, Poland \\ ${ }^{6}$ Future Processing, Gliwice, Poland \\ ${ }^{7}$ Laboratory of Imaging Diagnostics, Silesian Center for Heart Diseases in Zabrze, School of Medicine with the Division \\ of Dentistry in Zabrze, Poland \\ ${ }^{8}$ Chair and Institute of Medical Radiology and Radiodiagnostics in Zabrze, Medical University of Silesia in Katowice, Poland \\ ${ }^{9} 3^{\text {rd }}$ Chair and Clinical Department of Cardiology, School of Medicine with the Division of Dentistry in Zabrze, Medical \\ University of Silesia in Katowice, Silesian Center for Heart Diseases in Zabrze, Poland
}

Kardiochriugia i Torakochirurgia Polska 2017; 14 (3): 192-199

\begin{abstract}
This article discusses the protocol for myocardial perfusion scintigraphy performed with single-photon emission computed tomography (SPECT). Indications for SPECT are listed with consideration given to the results of the increasingly more common angio-CT examinations of the coronary arteries (multislice computed tomography). The paper also presents basic information about interpreting the results, including the scores of left ventricle myocardial perfusion using the 17-segment polar map, and explains the concept of total perfusion deficit. Key words: myocardial perfusion scintigraphy, coronary artery disease, bool eye imaging.
\end{abstract}

\section{Introduction}

Single-photon emission computed tomography (SPECT) is an imaging method that enables, among other things, the evaluation of myocardial perfusion. The source emitting the radiation is the patient, who is administered a radioactive tracer. The name tomography stems from Greek tomos, meaning "section" or "cut". Isotopic tomographic examination enables the determination of radiotracer accumulation in many sections. After the image is reconstructed, radiotracer activity is evaluated in sections along the short and long axis: the sagittal and frontal section.

\section{Streszczenie}

W pracy omówiono protokół scyntygrafii perfuzyjnej mięśnia sercowego wykonywanej za pomocą emisyjnej tomografii komputerowej pojedynczego fotonu (SPECT). Wymieniono wskazania z uwzględnieniem wyników coraz częściej wykonywanych badań angio-CT tętnic wieńcowych. Przedstawiono podstawowe informacje dotyczące interpretacji badania, w tym wskaźnika punktowej oceny perfuzji mięśnia sercowego lewej komory z uwzględnieniem podziału mapy polarnej na 17 segmentów, oraz wyjaśniono koncepcję wskaźnika całkowitego ubytku perfuzji.

Słowa kluczowe: scyntygrafia perfuzyjna serca, choroba wieńcowa, mapa polarna.

The main element of the gamma camera is the scintillator crystal, which, under the influence of gamma radiation, emits photons visible as flashes of light (i.e., scintillations; hence the phrase "single-photon" in the name of the examination). The registered light impulses are multiplied by photomultipliers and converted into electric current of various intensity. In this manner, the radiotracer's activity in the examined organ is visualized as a projection on a plane. The gamma camera, turning around the patient's chest and imaging the isotope's activity in many angular projections, enables the reconstruction of the 3D geometry of isotope activity within the heart. 
A SPECT-CT device is additionally equipped with a tomograph integrated with the gamma camera. CT enables reconstruction with attenuation correction, facilitating the elimination of false perfusion deficits resulting from the attenuation of emission by extracardiac tissues. This type of examination involves both transmission and emission as it consists in both a tomographic examination (exposition to $X$-ray radiation) and a second, main tomographic examination, superimposed over the first one, for imaging left ventricular perfusion (emission scintigraphy).

The imaging of cardiac perfusion involves the use of radiopharmaceuticals; their uptake by muscle cells is directly proportional to blood flow. The most frequently used radioactive tracer is methoxy-isobutyl-isonitrile (MIBI) labeled with technetium 99m ( $99 \mathrm{~m} \mathrm{Tc}-\mathrm{MIBI})$. Other radiotracers include tetrofosmin or thallium $\left({ }^{201} \mathrm{Tl}\right)$. The latter, hardly used in Poland, differs from the compounds labeled with technetium in that it undergoes redistribution in the myocardium. A one-time administration of ${ }^{201} \mathrm{Tl}$ at peak stress enables the performance of a one-day examination that will visualize radiotracer uptake during stress as well as rest because the radiotracer is redistributed after a while. However, the use of ${ }^{201} \mathrm{Tl}$ is not widespread for two main reasons. Firstly, it is obtained with the use of a cyclotron; secondly, its long half-life requires the use of appropriately smaller activities, which in turn translates into worse image quality. Some laboratories use mixed protocols using both ${ }^{201} \mathrm{Tl}$ and ${ }^{99 \mathrm{~m} T \mathrm{C}}$ [1]. Notwithstanding, most current perfusion investigations are performed with the use of non-redistributable compounds labeled with ${ }^{99 \mathrm{~m}} \mathrm{Tc}$. At the Silesian Center for Heart Diseases, the only radiopharmaceutical used for myocardial perfusion imaging is $99 \mathrm{~m} \mathrm{Tc}-\mathrm{MIBI}$, and the most frequently employed acquisition protocol is the two-day protocol.

Figure 1 presents the 2-day and the one-day protocol using radiopharmaceuticals labeled with ${ }^{99 \mathrm{~m}} \mathrm{Tc}$. In low-risk patients, a stress SPECT-CT examination with attenuation correction is performed first to exclude coronary artery disease. If the stress examination shows normal perfusion and muscle contractility, no additional resting examination is required, which significantly reduces the patient's exposition to radiation. In the remaining cases, the radiopharmaceutical must be administered twice.

\section{Indications for the examination}

The evaluation of cardiac perfusion using SPECT should be recommended to patients with an intermediate risk of coronary artery disease when other examinations evaluat-
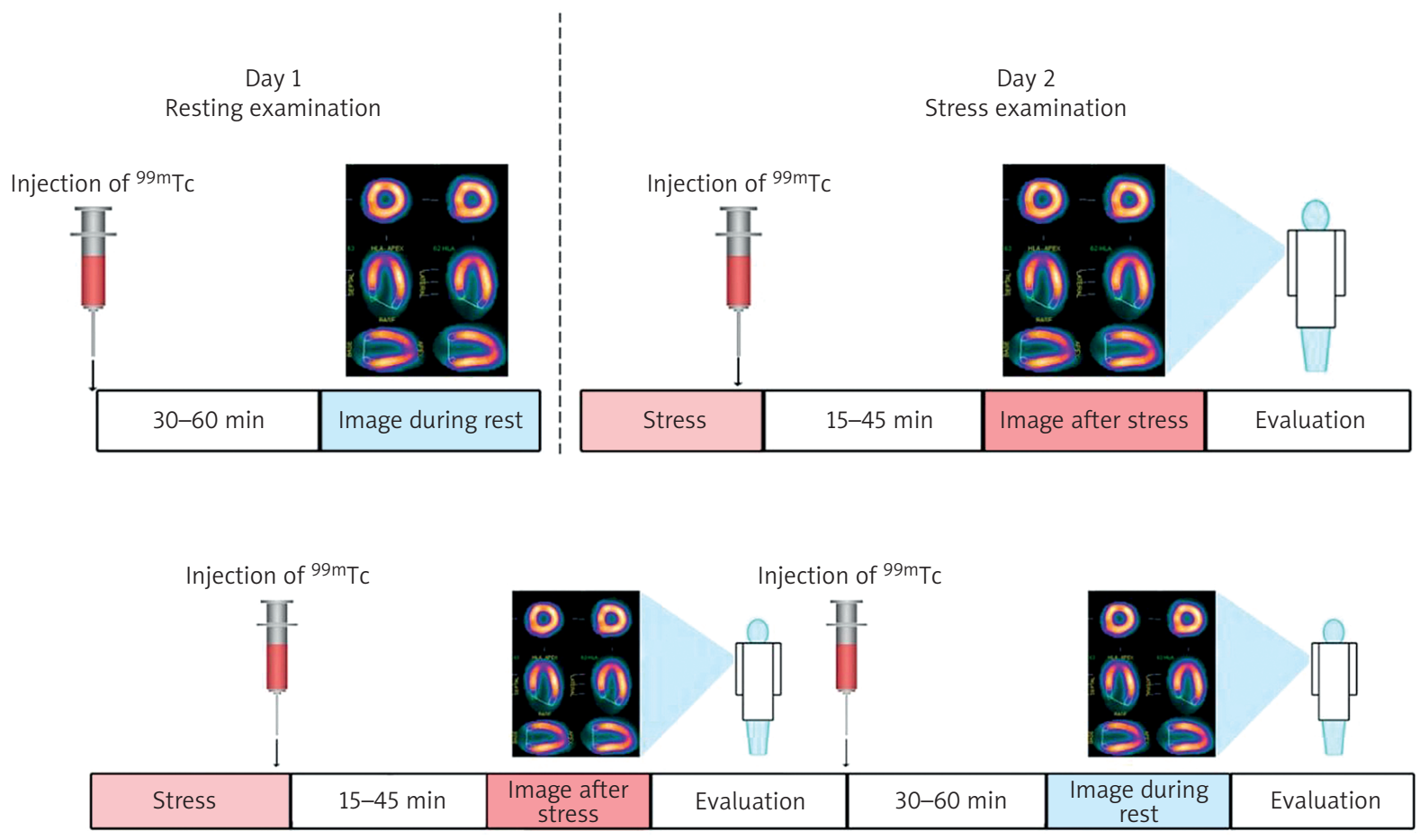

Fig. 1. Protocols for two-day (top) and one-day (bottom) examinations. The radiopharmaceutical labeled with $99 \mathrm{~m} T \mathrm{Tc}$ accumulates in cardiomyocytes proportionally to the flow of blood and is not subject to redistribution. The accumulation of the radiotracer reflects the flow of blood (myocardial perfusion) at the time when the radiotracer is intravenously injected. In order to obtain the image of cardiac perfusion during stress and rest, the radiotracer must be administered twice: at the peak of stress and during rest. In patients with a low risk of coronary artery disease, the initial examination should consist in stress scintigraphy. The two-day protocol is recommended in patients after coronary incidents or after percutaneous or surgical revascularization; in this case, the order in which the examinations are performed has little significance as the image has to be registered both under conditions of stress (exercise or pharmacological provocation with dobutamine or adenosine) and during rest. If the one-day protocol is employed, the first examination is usually the stress examination, with radiotracer dosage of approximately $8-10 \mathrm{mCi}$. After $3 \mathrm{~h}$, a resting examination is performed with a higher dose of the isotope $(24-30 \mathrm{mCi})$. Both the one-day and the two-day protocol can be considered to have equal diagnostic value; the choice which one to use depends on the preferences of the laboratory performing the investigation 
Tab. I. Parameters which can be assessed with myocardial perfusion scintigraphy using non-gated and ECG-gated examinations

\begin{tabular}{|c|c|}
\hline Non-gated perfusion examination & Gated perfusion examination \\
\hline Location, extent, and severity of perfusion disturbances at rest (scar) & $\begin{array}{l}\text { Ejection fraction, regional mobility and increased systolic thickening } \\
\text { of the left ventricular wall, end-systolic and end-diastolic volume } \\
\text { of the left ventricle, stroke volume, severity of contractility disorders } \\
\text { in stress tests - stunned myocardium }\end{array}$ \\
\hline $\begin{array}{l}\text { Location, extent, and severity of perfusion deficits during stress and } \\
\text { rest (reversible deficits - ischemia) }\end{array}$ & Diastolic function of the left ventricle \\
\hline $\begin{array}{l}\text { Other: transient left ventricular dilatation, right ventricular } \\
\text { hypertrophy }\end{array}$ & Phase analysis of systolic dyssynchrony, sphericity index \\
\hline
\end{tabular}

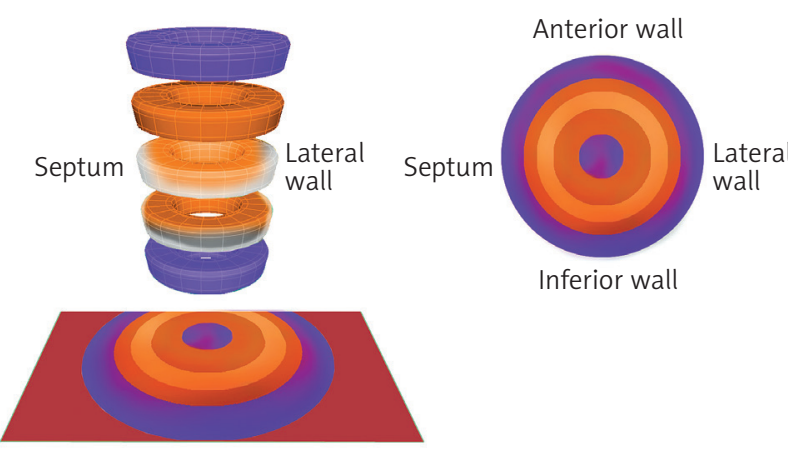

Fig. 2. The principles of constructing a polar map

ing the coronary reserve (such as electrocardiographic exercise testing or dobutamine stress echocardiography) yield ambiguous results. The examination can also be performed in patients with high indices of coronary artery calcification (CAC) revealed by $\mathrm{CT}$ in whom angio-CT with contrast was abandoned, patients in whom the evaluation of ostial lesions (especially in bifurcations) or coronary stents yields uncertain results, as well as in patients with borderline changes in order to assess their hemodynamic significance [2].

It should be noted that even in the case of patients with high CAC indices, the isotopic examination often does not reveal any significant perfusion disturbances; therefore, conducting invasive diagnostics in such cases is inexpedient. Performing perfusion scintigraphy in patients with high calcium scores can contribute to a significant reduction in the number of conventional coronary angiographies not ending with revascularization.

Another indication for performing a stress perfusion examination is myocardial bridging revealed by coronary angiography or angio-CT. In this case, the goal of the isotopic examination is to determine whether the systolic constriction of the vessel has any influence on left ventricular perfusion. In many cases, it turns out that the myocardial bridge does not cause ischemia [3].

Other uses for resting perfusion scintigraphy include the evaluation of myocardial vitality before revascularization procedures and differentiating ischemic cardiac injuries from injuries resulting from other causes. In these cases, a resting examination is sufficient.

Comparing the indications for perfusion scintigraphy and angio-CT examinations of the coronary arteries, it should be noted that, in contrast to tomography (which requires the administration of an iodic contrast agent), perfu- sion scintigraphy is not contraindicated in the presence of kidney failure.

In patients with steady heart rhythm, the count registration from the myocardial area is conducted synchronously in the individual phases of the cardiac cycle. In this mode of acquisition, the cardiac cycle is usually divided into 8 or 16 equal segments. Using ECG-gating in the examination enables the calculation of parameters that include the left ventricular ejection fraction, diastolic function parameters, as well as regional mobility and thickening of the left ventricular walls (interpreting the results of SPECT examinations using cardiac gating will be discussed in detail in the second part of this article). The option of phase analysis in gated examinations enables the quantitative evaluation of left ventricular systolic dyssynchrony in candidates for cardiac resynchronization therapy (Tab. I) [4].

\section{Evaluation of myocardial perfusion}

Depending on the type of myocardial perfusion disturbance, the examination may indicate post-MI scarring (when the resting examination shows reduced radiotracer uptake) or a reversible perfusion deficit (stress ischemia) when the radiotracer's activity is reduced during the stress examination, but not during the rest examination. In other situations, the examination may reveal post-MI scarring along with surrounding ischemia (resulting, e.g., from insufficient collateral circulation) or the presence of concurrent post-MI scarring (permanent perfusion deficit) in an area supplied by one vessel and ischemia (reversible deficit) in another area.

In each case when a perfusion disturbance is found, its location should be determined in relation to the segments affected by ischemia. For this purpose, it is useful to transform the 3D tomographic image of left ventricular perfusion into a polar map (Fig. 2). It is created as a result of imaging the distribution of radiotracer activity in two dimensions (Fig. 3). The model established by the American Heart Association (AHA) divides the polar map into 17 segments. The outermost segments correspond to the basal segments, and segment 17 represents the cardiac apex (Fig. 4).

Computer software for the quantitative assessment of perfusion disturbances enables the comparison of the scintigraphic images obtained from the patient with a database containing averaged data from healthy individuals. The perfusion deficit on the polar map is evaluated with re- 


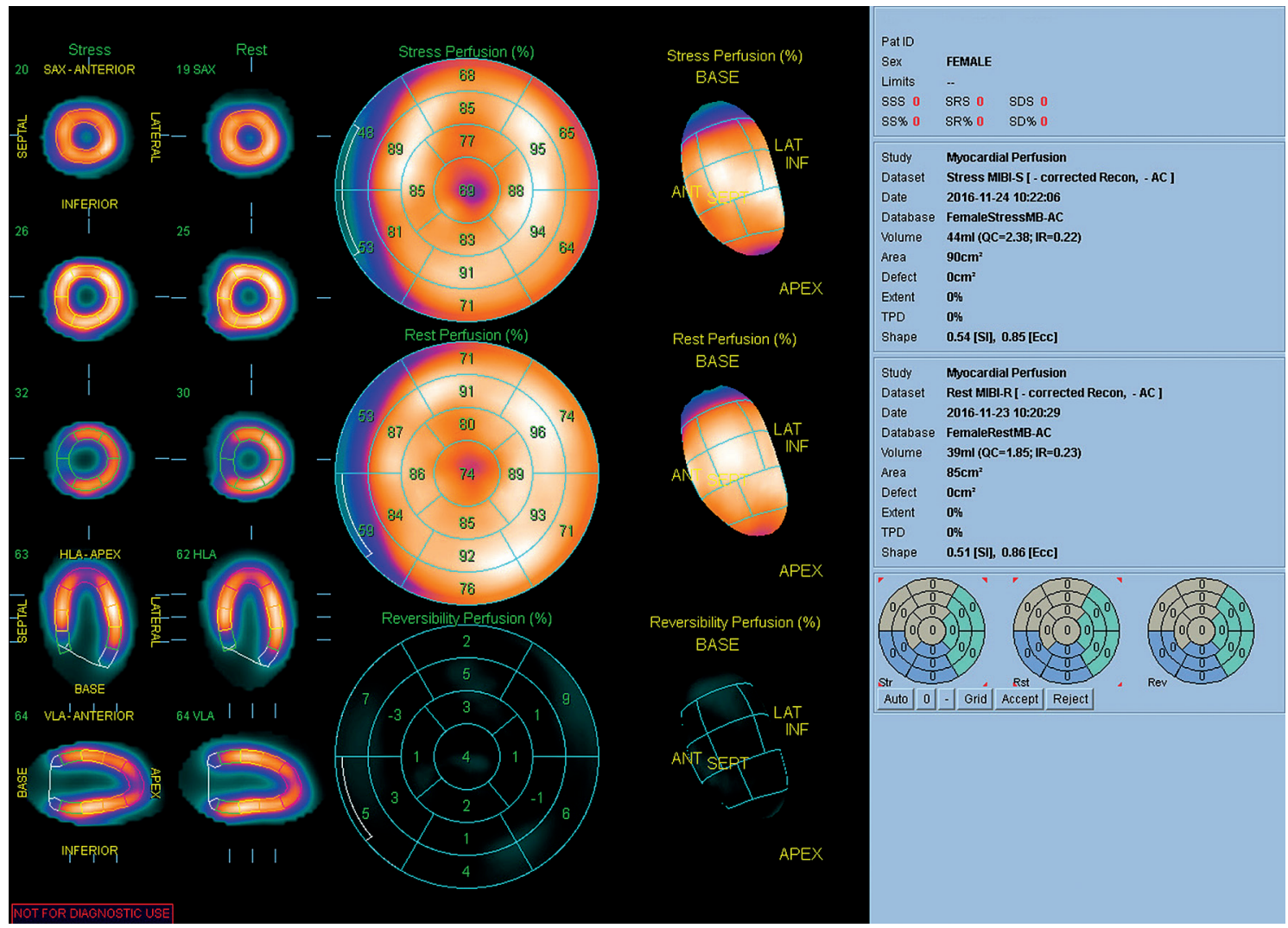

Fig. 3. Normal results of myocardial perfusion scintigraphy. Middle: a normal polar map of left ventricular perfusion with noticeably lower radiotracer activity on the circumference of the basal segments and, to a lesser degree, in the apex. Top right: stress and rest perfusion scores (in this case amounting to 0 points). Bottom right: the polar map divided into 17 segments. The examination was performed in the Laboratory of Nuclear Medicine of the Silesian Center for Heart Diseases in Zabrze (Cedars-Sinai Medical Center software; QPS - Quantified Perfusion Spect)

gard to its extent (Fig. 5). For this purpose, all areas on the polar map that contain values below the assumed threshold are blackened (blackout polar map). The extent of the deficit is calculated in relation to the surface of the middle layer, omitting the valve output in the left ventricular outflow tract, and is expressed in $\mathrm{cm}^{2}$ or percentages. The second parameter describing perfusion deficit is the severity of radiotracer uptake reduction in relation to standard deviation (Fig. 6). In turn, the parameters that pertain to both the extent and severity of perfusion deficits are the segmental myocardial perfusion score (five-point scale; Tab. II) and the Total Perfusion Deficit (TPD) described below $[5,6]$.

\section{Scoring left ventricular myocardial perfusion during rest and stress using 17 segments}

Computer software for the semiquantitative evaluation of left ventricular perfusion is used to calculate the perfusion score considering both the extent and severity of ischemia in relation to the 17 segments of the polar map (Figs. 5 and 6). Normal perfusion (as compared with averaged gender-specific data from the population of healthy individuals) is indicated on the scale as a score of 0 (normal perfusion in relation to the control group). Mild and moderate perfusion impairment is indicated by 1 and 2 points, respectively. A score of 3 points indicates significant perfu- sion impairment, while a score of 4 points is used to indicate total impairment, meaning practically no perfusion (Tab. II).

An example of scoring perfusion using the 17-segment model of the left ventricle is presented on Figure 7. It is estimated that a left ventricular perfusion deficit with a score of 1 or 2 indicates that isotopic activity in this segment is approximately $60 \%$ in comparison to the area of radiotracer accumulation specified as $100 \%$. Three points indicate radiotracer activity between $40 \%$ and $60 \%$, while 4 points indicate radiotracer activity below $40 \%$ in relation to the area with $100 \%$ activity. Scoring perfusion disturbances in resting examinations is also useful for evaluating myocardial vitality.

The global scoring of myocardial perfusion uses measures such as: the Summed Stress Score (SSS), the Summed Rest Score (SRS), and the Summed Difference Score (SDS). The SSS is the sum of the individual scores from the $17 \mathrm{seg}$ ments of the polar map obtained during stress. When the SSS amounts to less than 4, the perfusion is considered normal or minimally abnormal (no significant perfusion disturbances); a result of 4-8 points indicates mildly abnormal perfusion; 9-13 - moderately abnormal perfusion; and 13 or more - the presence of significant extensive ischemia (Tab. III). 

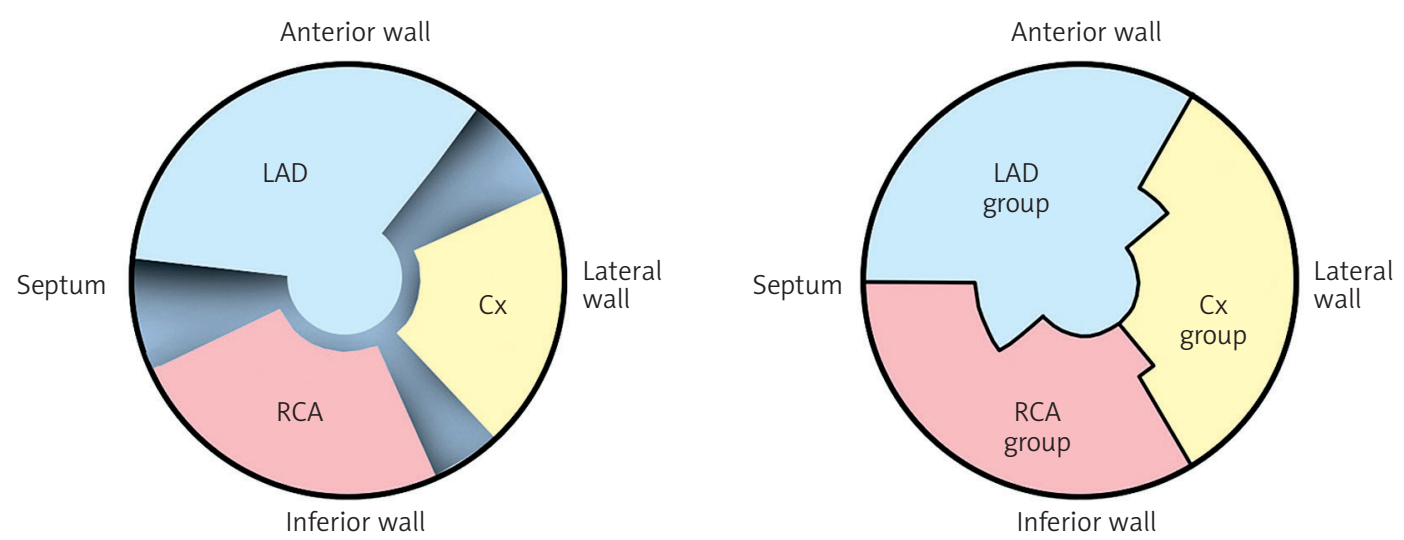
(1) Basal anterior
(2) Basal anteroseptal
(3) Basal inferoseptal
(4) Basal inferior
(5) Basal inferolateral
(6) Basal anterolateral
(7) Mid anterior
(8) Mid anteroseptal
(9) Mid inferoseptal

(10) Mid inferior

(11) Mid inferolateral

(12) Mid anterolateral

13) Apical anterior

14 Apical septal

(15) Apical inferior

(16) Apical lateral

(17) Apex

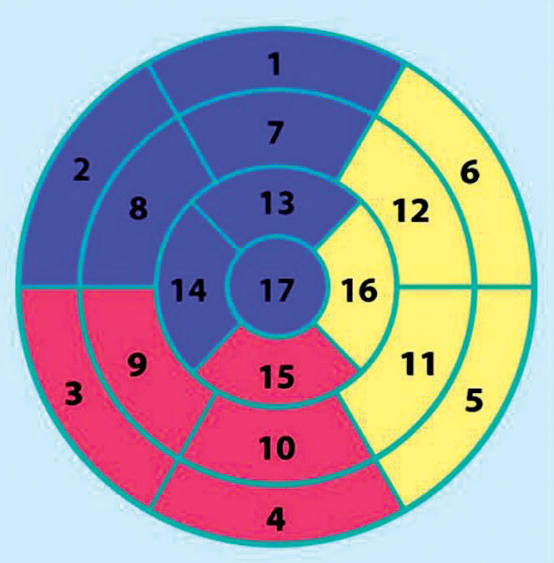

Fig. 4. Polar map division according to Cedars-Sinai Medical Center software. Top left: polar map division based on arteries; top right: division based on coronary artery groups. In the polar map divided according to arteries, the areas which cannot be unequivocally assigned to the supply area of a particular coronary vessel due to variable vascularization are blackened. LAD - left anterior descending branch, Cx - circumflex branch, RCA - right coronary artery. Bottom: left ventricular polar map division into 17 segments according to the American Heart Association. The order in which the segments are numbered is counterclockwise. Basal segments are numbered 1-6, midle segments: 7-12, and paraapical segments: 13-16. The figure also assigns the segments to particular coronary artery groups. The area assigned to the left anterior descending artery and its branches is marked with blue; the group of segments assigned to the circumflex artery and its branches is marked with yellow, and the right coronary artery group is marked with red. It should be noted that the assignment of the polar map segments to specific coronary arteries is approximate and schematic; it may vary depending on the type of left ventricular vascularization

With regard to the number of segments with abnormal perfusion, the ischemic area is described as small when it involves 1 or 2 segments, moderate when it involves 3 or 4 segments, or large when it involves 5 or more segments.

\section{SDS - the difference between the SSS and the SRS}

SDS can be calculated by subtracting the SRS from the SSS (SDS = SSS - SRS). This measure is used to describe the degree to which the deficit/ischemia is reversible. An SDS score of 0-1 indicates no ischemia; $2-4$ points indicate mild ischemia; 5-6 points indicate moderate ischemia; while 7 or more points indicate severe ischemia, i.e., significant stress perfusion deficit (Tab. III).

\section{Percentage measures of left ventricular perfusion (SS\%, SR\%, and SD\%)}

The fact that the polar map used to be divided into 20 segments, while the currently recommended division includes 17 segments, necessitated the introduction of normalized SSS, SRS, and SDS measures, i.e., respectively, SS\%, SR\%, and SD\%. SS\% is calculated by dividing the SSS by a number corresponding to the SSS value indicating a deficit of 4 in every segment (80 points for 20 segments or 68 points for 17 segments) and then multiplying the result by $100 \%$. For example, when using the 17-segment division, the SS\% for a SSS of 13 amounts to $19 \%$ (13: $68 \times 100 \%)$. SS\% values up to $4 \%$ indicate normal perfusion; $5-9 \%-$ mild abnormality; 10-14\% - moderate abnormality; $14 \%$ or 


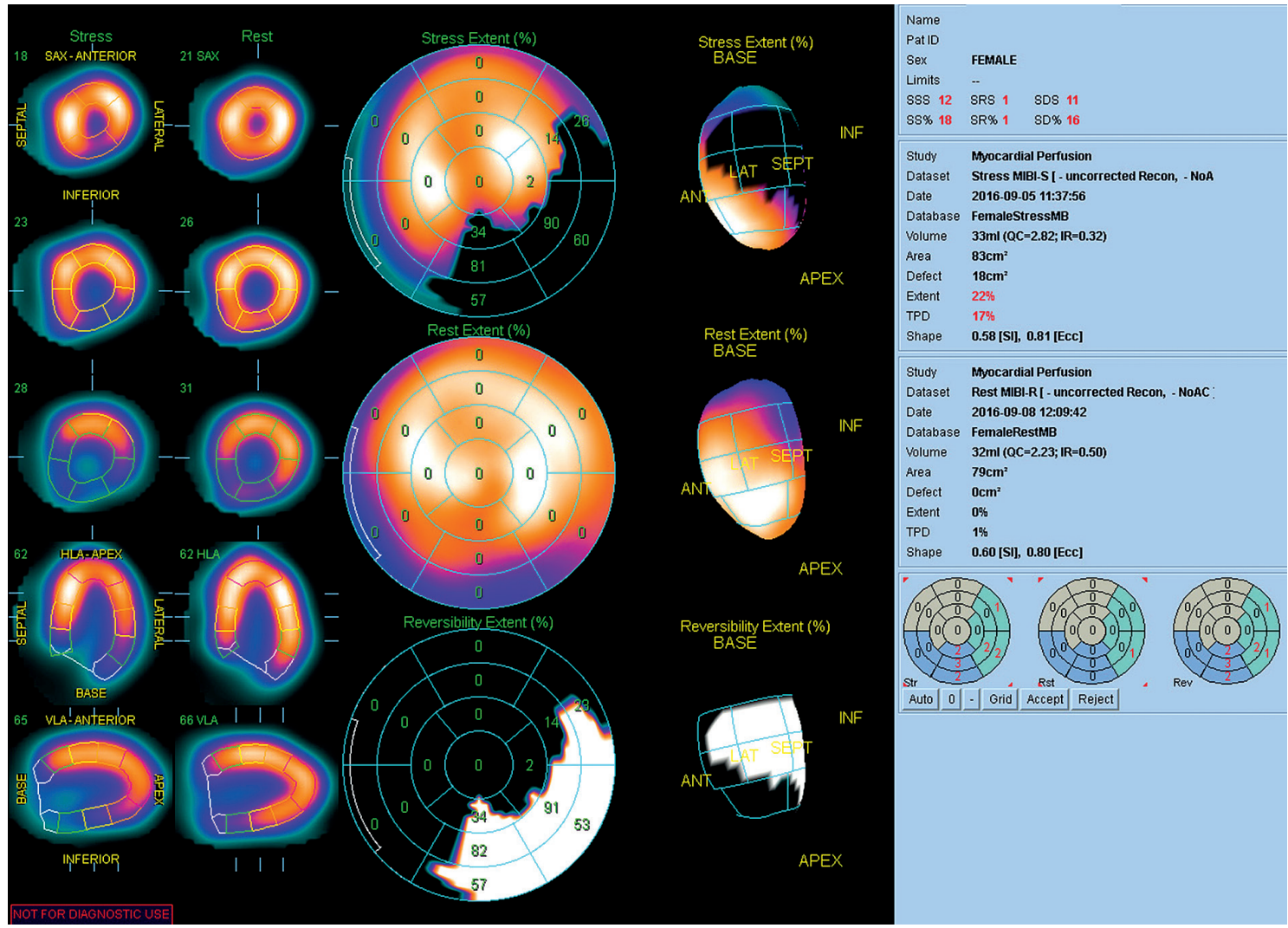

Fig. 5. The figure presents the extent of a stress perfusion deficit. All the areas on the polar map with values below normal are blackened (blackout polar map). The perfusion deficit involves the inferolateral wall, extending over $18 \mathrm{~cm}^{2}$, i.e., $22 \%$ of the left ventricular surface. The ischemic area may correspond to the right coronary artery or the circumflex branch. The examination was performed in the Laboratory of Nuclear Medicine of the Silesian Center for Heart Diseases in Zabrze (Cedars-Sinai Medical Center software; QPS - Quantified Perfusion Spect)

more - significant abnormality (Tab. III). The SR\% and SD\% values are calculated in the same manner. Patients with SD\% exceeding $10 \%$ are believed to benefit from revascularization regardless of their left ventricular ejection fraction [7]. In turn, optimal pharmacological therapy accompanied by changes in lifestyle is preferred in patients with SD\% smaller than $10 \%[8,9]$.

\section{Total perfusion deficit}

Total perfusion deficit (TPD) is calculated based on both the extent and severity of ischemia. As shown in Figure 8 , the area below the lower margin of the normal values of the activity profile, but above the curve of the circular activity profile for a given slice shows the perfusion deficit for this slice [10]. The individual TPD values are calculated for all circular activity profiles of the myocardium and are added together as the total deficit of perfusion. The TPD is equivalent with the polar map's segmental perfusion score, but it differs in that it is a constant value that does not pertain to the individual segments. The established normal values of TPD fall below 5\%; TPD of 5-9\% indicates slight abnormality; $10-14 \%$ - moderate abnormality; and 15\% or more - significant abnormality (Tab. III).

\section{Transient left ventricular dilatation and right ventricular radiotracer uptake}

During the evaluation of perfusion results, one should consider transient ischemic dilatation of the left ventricle, a phenomenon that occurs in the course of multivessel coronary artery disease with balanced and generalized subendocardial ischemia affecting all walls of the left ventricle. Its occurrence should be treated as a positive result and an indication for conventional coronary angiography even in the absence of significant perfusion deficits.

Another abnormality that should be considered is the accumulation of the radiotracer in the right ventricle. Normally, the right ventricle is almost invisible in a scintigraphic examination; increased radiotracer uptake indicates right ventricular hypertrophy.

\section{Assessing the risk of myocardial infarction or death due to cardiac causes based on the perfusion score}

The presence and intensity of stress ischemia determines the management. If significant stress ischemia is revealed, the patient should be referred for conventional coronary angiography with revascularization in mind (SD\% 


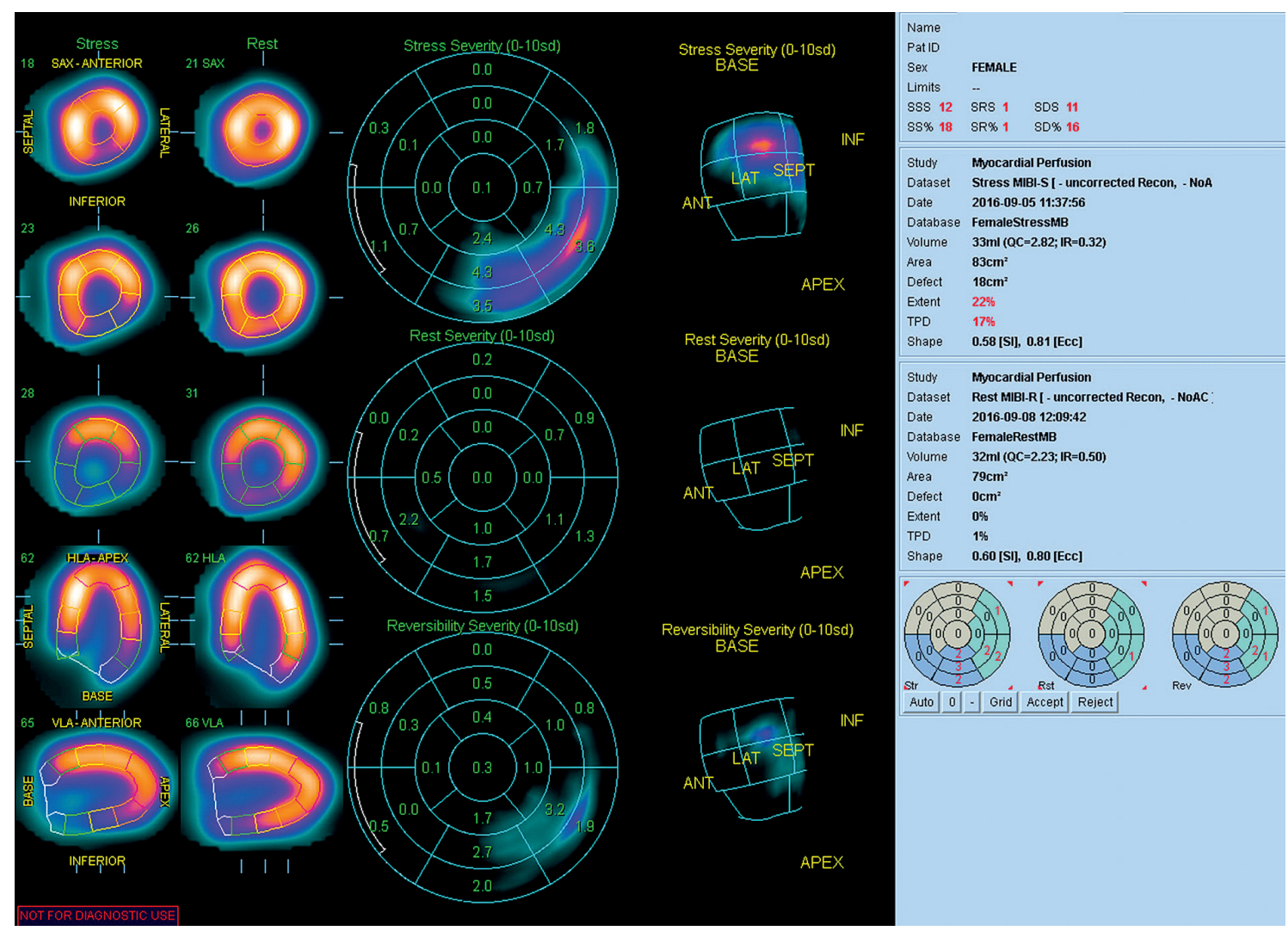

Fig. 6. The figure presents the severity of a stress perfusion deficit (in the same patient as in Fig. 5). The most severe perfusion deficit is present in segment 5 (the inferolateral basal segment according to AHA), and the artery responsible for the ischemia is most likely the right coronary artery. The examination was performed in the Laboratory of Nuclear Medicine of the Silesian Center for Heart Diseases in Zabrze (QPS software)

Tab. II. A five-point scale for scoring myocardial perfusion

\begin{tabular}{lc} 
Perfusion & Score \\
Normal & 0 \\
\hline Minimal, mild impairment of perfusion, ambiguous image & 1 \\
\hline Moderate impairment of perfusion & 2 \\
\hline Significant impairment of perfusion & 3 \\
\hline No perfusion & 4 \\
\hline
\end{tabular}

>10). If the ischemic area after exercise is sufficiently small, conservative treatment is a reasonable alternative $[8,9]$.

In the context of the 17-segment model of the left ventricle, when the SSS falls between 0 and 3 (normal/minimally abnormal result), the risk of myocardial infarction or cardiac death is minimal; when the SSS falls between 4 and 7 (slightly abnormal result), the risk is low; when the SSS is between 8 and 12 , the risk is considered intermediate;
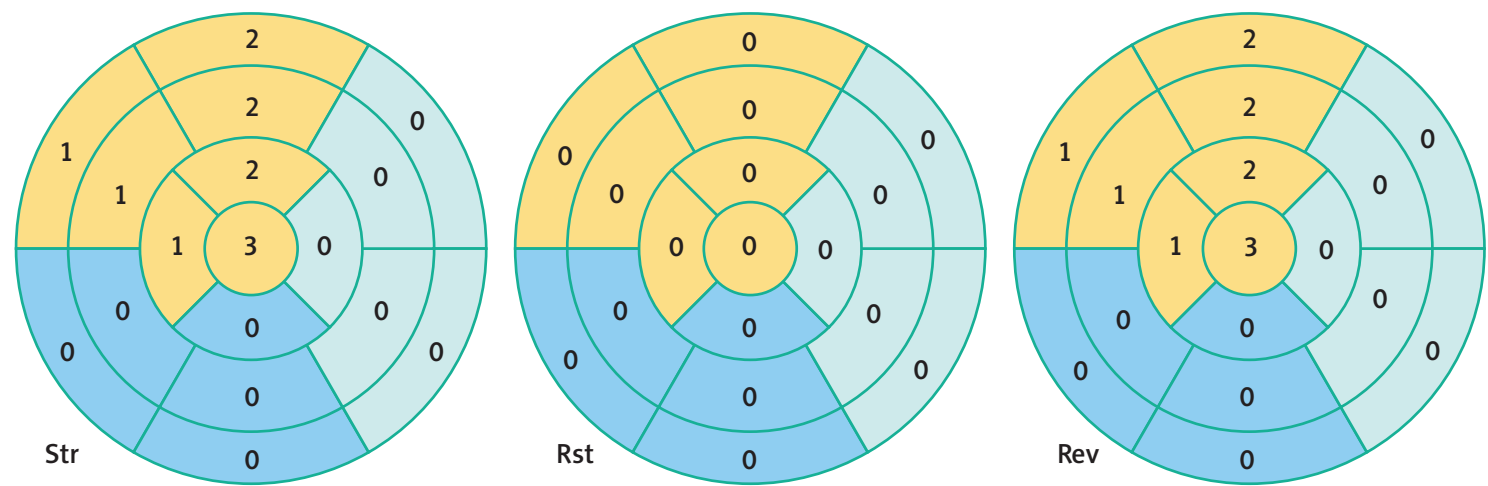

SSS - Summed Stress Score, SRS - Summed Rest Score, SDS - Summed Difference Score, Str - stress, Rst - rest, Rev - reversibility

Fig. 7. An example of scoring myocardial perfusion. In this case, the summed score of perfusion deficits amounted to 12 during stress (SSS) and 0 during rest (SRS). Therefore, the difference (SDS) amounted to 12 points. The perfusion deficit is located within the anterior wall, septum, and apex (supply area of the left anterior descending branch). Due to the number of segments affected by stress perfusion deficits, the examination result is significantly positive 
Tab. III. Interpreting left ventricular perfusion scores

\begin{tabular}{|c|c|c|c|c|}
\hline SSS & SS\% & SDS & TPD (\%) & Result \\
\hline$<4$ & $<5$ & $<2$ & $<5$ & $\begin{array}{l}\text { Normal or minimally } \\
\text { abnormal result }\end{array}$ \\
\hline $4-8$ & $5-9$ & $2-4$ & $5-9$ & Mildly abnormal result \\
\hline $9-13$ & $10-14$ & $5-6$ & $10-14$ & Moderately abnormal result \\
\hline$>13$ & $>14$ & $>6$ & $>14$ & Significantly abnormal result \\
\hline
\end{tabular}

SSS - summed stress score, SS\% - summed stress percentage, SDS - summed difference score, TPD - total perfusion deficit (percentage)

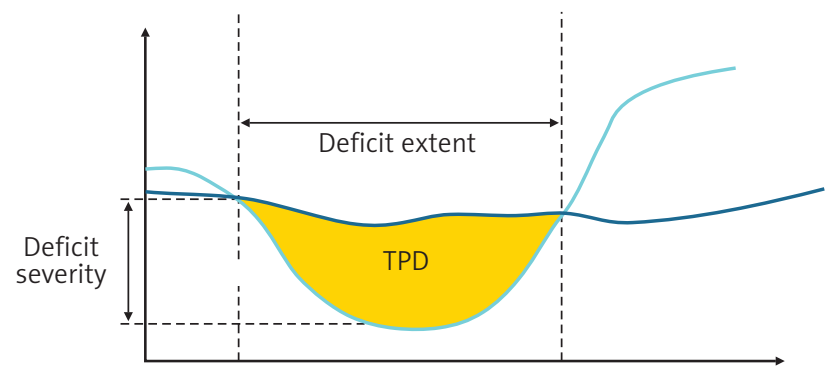

Fig. 8. Illustration of the concept of Total Perfusion Deficit for a single cardiac segment. The light blue curve shows the circular activity profile, the dark blue curve shows the lower margin of the normal values of the activity profile

and when the SSS exceeds 12 points, the risk is high [11]. Other authors, using the 20-slice model of the left ventricular polar map, assess the risk as low for SSS values between 4 and 8, intermediate for SSS values between 9-13, and high for SSS values exceeding 13 [12]. A meta-analysis encompassing over 12,000 patients demonstrated that the risk of myocardial infarction or death amounted to $0.6 \%$ for patients with normal stress scintigraphy results and $7.4 \%$ for patients with abnormal results [13]. In another study, the annualized mortality rate in a group of 473 patients with normal results of stress cardiac SPECT examinations using Tc-99m-MIBI was only $0.2 \%$ over 3 years [14].

\section{Conclusions}

The proper interpretation of cardiac perfusion scintigraphy using the SPECT method not only enables the diagnosis of significant stress ischemia, but also provides valuable prognostic information determining the choice of treatment (optimal pharmacological therapy vs. invasive treatment) to be provided to patients, including those with borderline changes revealed by angio-CT or with high indices of coronary artery calcification.

\section{Disclosure}

Authors report no conflict of interest.

\section{References}

1. Makita A, Matsumoto N, Suzuki Y, Hori Y, Kuronuma K, Yoda S, Kasama S, Iguchi N, Suzuki Y, Hirayama A. Clinical feasibility of simultaneous acquisition rest $(99 \mathrm{~m}) \mathrm{Tc} / \mathrm{stress}$ (201)Tl dual-isotope myocardial perfusion single- photon emission computed tomography with semiconductor camera. Circ 2016; 80: 689-695.

2. Wasilewski J, Głowacki J, Gąsior M, Zembala M. Komentarz do dokumentu ACCF/ACR/AHA/NASCI/SAIP/SCAI/SCCT 2010 Expert Consensus Document on Coronary Computed Tomographic Angiography. A Report of the American College of Cardiology Foundation Task Force on Expert Consensus Documents. Daniel B. Mark, Daniel S. Berman, Matthew J. Budoff, J. Jeffrey Carr, Thomas C. Gerber, Harvey S. Hecht, Mark A. Hlatky, John McB. Hodgson, Michael S. Lauer, Julie M. Miller, Richard L. Morin, Debabrata Mukherjee, Michael Poon, Geoffrey D. Rubin, and Robert S. Schwartz. J Am Coll Cardiol 2010; 55: 2663-2699. Kardiochir Torakochir Pol 2010; 7: 249-251.

3. Tarantini G, Migliore F, Cademartiri F, Fraccaro C, lliceto S. Left anterior descending artery myocardial bridging: a clinical approach. J Am Coll Cardiol 2016; 68: 2887-2899.

4. Chen J, Garcia EV, Bax JJ, Iskandrian AE, Borges-Neto S, Soman P. SPECT myocardial perfusion imaging for the assessment of left ventricular mechanical dyssynchrony. J Nucl Cardiol 2011; 18: 685-694.

5. Yoda S, Nakanishi K, Tano A, Hori Y, Suzuki Y, Matsumoto N, Hirayama A. Validation of automated quantification of nuclear cardiology in Japanese patients using total perfusion deficits: comparison with visual assessment. J Cardiol 2014; 63: 350-357.

6. Berman DS, Kang X, Gransar H, Gerlach J, Friedman JD, Hayes SW, Thomson LE, Hachamovitch R, Shaw LJ, Slomka PJ, Yang LD, Germano G. Quantitative assessment of myocardial perfusion abnormality on SPECT myocardial perfusion imaging is more reproducible than expert visual analysis. J Nucl Cardiol 2009; 16: 45-53.

7. Hachamovitch R, Rozanski A, Hayes SW, Thomson LE, Germano G, Friedman JD, Cohen I, Berman DS. Predicting therapeutic benefit from myocardial revascularization procedures: are measurements of both resting left ventricular ejection fraction and stress-induced myocardial ischemia necessary? J Nucl Cardiol 2006; 13: 768-778.

8. Shaw LJ, Hage FG, Berman DS, Hachamovitch R, Iskandrian A. Prognosis in the era of comparative effectiveness research: where is nuclear cardiology now and where should it be? J Nucl Cardiol 2012; 19: 1026-1043.

9. Hachamovitch R, Hayes SW, Friedman JD, Cohen I, Berman DS. Comparison of the short-term survival benefit associated with revascularization compared with medical therapy in patients with no prior coronary artery disease undergoing stress myocardial perfusion single photon emission computed tomography. Circulation 2003; 107: 2900-2907.

10. Slomka PJ, Nishina H, Berman DS, Akincioglu C, Abidov A, Friedman JD, Hayes SW, Germano G. Automated quantification of myocardial perfusion SPECT using simplified normal limits. J Nucl Cardiol 2005; 12: 66-77.

11. Dvorak RA, Brown RK, Corbett JR. Interpretation of SPECT/CT myocardial perfusion images: common artifacts and quality control techniques. Radiographics 2011; 31: 2041-2057.

12. Pazhenkottil AP, Ghadri JR, Nkoulou RN, Wolfrum M, Buechel RR, Küest SM, Husmann L, Herzog BA, Gaemperli O, Kaufmann PA. Improved outcome prediction by SPECT myocardial perfusion imaging after CT attenuation correction. J Nucl Med 2011; 52: 196-200.

13. Iskander S, Iskandrian AE. Risk assessment using single photon emission computed tomography technetium-99m sestamibi imaging. J Am Coll Cardiol 1998; 32: 57-62.

14. Soman P, Parsons A, Lahiri N, Lahiri A. The prognostic value of normal Tc-99m-sestamibi SPECT study in suspected coronary artery disease. J Nucl Cardiol 1999; 6: 252-256. 